\title{
Extreme-ultraviolet and hard X-ray signatures of electron acceleration during the failed eruption of a filament ${ }^{\star}$
}

\author{
A. Netzel ${ }^{1}$, T. Mrozek ${ }^{1,2}$, S. Kołomański ${ }^{1}$, and S. Gburek ${ }^{2}$ \\ 1 Astronomical Institute, University of Wrocław, ul. Kopernika 11, 51-622 Wrocław, Poland \\ e-mail: mrozek@astro.uni.wroc.pl \\ 2 Space Research Centre, Polish Academy of Sciences, Solar Physics Division, ul. Kopernika 11, 51-622 Wrocław, Poland \\ Received 12 March 2012 / Accepted 14 September 2012
}

\section{ABSTRACT}

\begin{abstract}
Aims. We search for extreme-ultraviolet (EUV) brightenings in TRACE $171 \AA$ A images and hard X-ray (HXR) bursts observed during failed eruptions. We expect that if an eruption is confined by interaction with overlaying magnetic structures, we should observe effects caused by reconnection between magnetic structures and acceleration of particles.

Methods. We used TRACE observations of three well-observed failed eruptions. A semi-automated method was used to search for abrupt brightness changes in the TRACE field of view. The EUV images were compared to the HXR spatial distribution reconstructed from YOHKOH/HXT and RHESSI data. The EUV light curves of a selected area were compared to height profiles of eruption, HXR emission, and the HXR photon spectral index of a power-law fit to the HXR data.

Results. We have found that EUV brightenings are closely related to the eruption velocity decrease, to HXR bursts, and to episodes of hardening of the HXR spectra. The EUV-brightened areas are observed far from the flaring structure, in footpoints of large systems of loops observed 30-60 min after the maximum of a flare. These are not "post-flare" loops, which are also observed, but at significantly lower heights. The high-lying systems of loops are observed at heights equal to the height at which the eruption was observed to stop. We observed only one HXR source that was spatially correlated with EUV brightening. For other EUV-brightened areas we estimated the expected brightness of HXR sources.

Conclusions. We find that EUV brightenings are produced by interaction between the erupting structure with overlaying loops. The interaction is strong enough to heat the system of high loops. These loops cool and are visible in the EUV range about 30-60 min later. The estimated brightness of HXR sources associated with EUV brightenings shows that they are too weak to be detected with present instruments. However, next-generation instruments will have sufficient dynamic range and sensitivity to enable such observations.
\end{abstract}

Key words. Sun: flares - Sun: UV radiation - Sun: filaments, prominences - Sun: X-rays, gamma rays

\section{Introduction}

The solar corona is filled with plasma frozen in coronal magnetic lines. Structures formed in that way can be quiet or dynamic. Perfect examples of such structures are coronal loops or filaments (prominences). These features can vary in size (up to $10^{6} \mathrm{~km}$ for giant arches) and in temperature ( $\geq 10 \mathrm{MK}$ for postflare loops). Sometimes they undergo dynamic changes that we believe are due to magnetic reconnection. These changes release great amount of energy leading to the most energetic events in our solar system like filaments eruptions, flares and coronal mass ejections.

Gilbert et al. (2007) proposed a classification for filament eruptions based on the different amount of magnetic structure and erupted mass. The three proposed groups are 1. Full - most $(\geq 90 \%)$ of the filament mass and magnetic structure erupted; 2. Partial - divided into two classes A and B. Class A is the eruption of the entire magnetic structure with little or even no mass. Class B is the partial eruption of magnetic structure with some or no mass; 3. Failed - neither mass nor magnetic structure escapes from the Sun. Flares can be classified in a similar way. Solar flares associated with coronal mass ejections (CMEs) are the eruptive flares, while flares that occur without CMEs are confined flares (Sakai \& de Jager 1996).

$\star$ Movies are available in electronic form at http://www . aanda . org
There are several possible mechanisms proposed that may lead to failed eruptions. Among them is kink instability (Török \& Kliem 2005), reaching an upper equilibrium (Vršnak 2001; Green et al. 2002), forces within an erupting flux rope (Vršnak 1990), magnetic tension force and momentum exchange with the background plasma (Wang \& Sheeley 1992; Archontis \& Török 2008), insufficient energy released in the low corona (Shen et al. 2011), and confinement by the overlaying coronal magnetic field (Hirose et al. 2001; Wang \& Zhang 2007; Liu 2008; Mrozek 2011)

The confining mechanism based on interaction with the overlaying coronal magnetic field was explored theoretically and observationally. The theoretical simulation carried out by Amari \& Luciani (1999) showed that erupting magnetic flux during its evolution can reconnect with the overlaying arcade and remain confined. Numerical experiments showed that evolution of a flux rope (and the whole flux system) after its emergence is dependent on the ambient magnetic field (Archontis \& Török 2008). Magnetohydrodynamics (MHD) simulations of a confined filament eruption on 27 May 2002 carried out by Török \& Kliem (2005) showed that the decrease of the overlaying magnetic field with height is a key factor leading to the failed eruption. Similar results have been reported by Wang \& Zhang (2007), who examined $104 \mathrm{X}$-class flares and found that $10 \%$ of them were confined. The confined events were observed closer to the center of an active region where the overlaying coronal magnetic field is the strongest. 
Table 1. Analyzed events.

\begin{tabular}{|c|c|c|c|c|c|c|c|c|c|}
\hline \multirow[t]{2}{*}{ No. } & \multirow[t]{2}{*}{ Event } & \multirow[t]{2}{*}{ Coordinates } & \multicolumn{4}{|c|}{ Associated flare } & \multicolumn{2}{|c|}{ Eruption } & \multirow[t]{2}{*}{$\mathrm{CMB}$} \\
\hline & & & GOES class & Start & Max & End & Start & Max & \\
\hline 1 & SOL1999-10-22T09:16 & N20W76 & $\mathrm{C} 4.8$ & 09:10 & 09:16 & $09: 29$ & 09:11 & 09:15 & No \\
\hline 2 & SOL2004-07-14T05:23 & N12W62 & M6.2 & 05:02 & $05: 23$ & $05: 27$ & 05:19 & $05: 25$ & No \\
\hline 3 & SOL2004-08-13T18:12 & S13W24 & $\mathrm{X} 1.0$ & 18:07 & $18: 12$ & $18: 15$ & 18:11 & $18: 23$ & Yes \\
\hline
\end{tabular}

Recently, Mrozek (2011) analyzed one failed eruption that was stopped by interaction with the overlaying coronal magnetic field. The interaction was observed as an unusual behavior of the eruption front. It was observed to change its shape, and fall back, and side eruptions occurred subsequently. Moreover, radial oscillations of the loops that were pushed to greater heights by the interaction with the eruption were observed. The extremeultraviolet (EUV) brightenings were observed in the foot points of the overlaying magnetic structure during the deceleration of the eruption. These brightenings may be produced by nonthermal electrons accelerated during the episodes of interaction between the eruption and the overlaying magnetic field. However, hard X-ray (HXR) sources spatially correlated with the EUV brightenings were not found. The eruption occurred in the active region with a clear quadruple configuration. Flares and accompanied eruptions in this magnetic configuration were theoretically modeled by Uchida et al. (1999), and Hirose et al. (2001). In this model an eruption may interact with the overlaying magnetic field and may eventually be stopped.

Assuming that during the evolution of the eruption there are episodes of interaction with the overlaying field, we can expect some signatures of nonthermal electrons. If nonthermal electrons occur in large overlaying magnetic loops their signatures will be observed at some distance from the flare and the associated eruption. In this paper we present a qualitative analysis of the three well-observed failed eruptions. The energy balance and quantitative analysis will be presented in a follow-up paper.

\section{The observations}

In our investigation we used EUV data from the TRACE satellite (Handy et al. 1999) and HXR data obtained by YOHKOH/HXT (Kosugi et al. 1991) and RHESSI (Lin et al. 2002). TRACE was operated from April 2, 1998 to June 21, 2010. The telescope was equipped with a set of filters that enabled us to observe the Sun in several wavelengths covering a temperature range from tens of thousands of Kelvins up to $2 \mathrm{MK}$. The $30 \mathrm{~cm}$ telescope and $1024 \times 1024$ CCD detector recorded images with a 1 -arcsec resolution ( 0.5 arcsec pixel size). The typical cadence for images obtained in the same filter was in the range of 2-40 s. We used images obtained with the $171 \AA$ filter, which is sensitive to $1 \mathrm{MK}$ plasma with a very small addition of a hot (10 MK) component (Phillips et al. 2005).

The HXR data from YOHKOH/HXT and RHESSI were used to obtain images and spectra. YOHKOH/HXT was a Fouriersynthesis imager equipped with a collimator and a detector plate. The collimator consisted of the grid plates at both ends. The sixty-four grids with different slit widths and orientations provided detectors with different Fourier components of the actual HXR sources distribution. The detectors measured HXR signals in four energy channels: L (14-23 keV), M1 (23-33 keV), M2 (33-53 keV), and H (53-93 keV). Images can be reconstructed with the MEM or PIXON algorithms. We used the PIXON algorithm. The spatial resolution is equal to $5^{\prime \prime}$. The best time-resolution is $0.5 \mathrm{~s}$, but in practice it is worse and depends on count statistics. Since HXR signals were measured in four energy bands, it is possible to perform a spectral analysis. The lowest channel, $\mathrm{L}$, is affected by thermal radiation and should be excluded from an analysis of the nonthermal component.

RHESSI data are available for events Nos. 2 and 3 (Table 1). RHESSI is a Fourier-synthesis imager that produces modulation of incoming HXR radiation by rotating the whole instrument. The rotation period of RHESSI is close to $4 \mathrm{~s}$. Images can be reconstructed with several different algorithms (Hurford et al. 2002). Here we used the PIXON algorithm (Metcalf et al. 1996). The time resolution for imaging is limited by the rotation period. The spatial resolution depends on the grids chosen for reconstruction and on the reconstruction algorithm. In practice the spatial resolution is rarely higher than $7^{\prime \prime}$. RHESSI uses large germanium detectors to measure time and energy of each detected HXR photon (Smith et al. 2002). The energies are measured from $3 \mathrm{keV}$ ( $1 \mathrm{keV}$ energy resolution) to several $\mathrm{MeV}$ ( $5 \mathrm{keV}$ resolution). Thus, the instrument provides us with highenergy resolution spectra, which allows performing a detailed spectral analysis.

The failed eruptions are hard to find with automated methods. The main reasons are the following. (1) The TRACE EUV images are full of artificial sources such as particle tracks, diffraction structures, etc. (2) A failed eruption changes its shape very fast and may change the direction of the expansion - the temporal resolution of TRACE images is not sufficient to analyze these changes. (3) The braking of the eruption may be smooth or abrupt and may be observed along the whole front or only in its part. For these reasons we visually inspected the TRACE movies database ${ }^{1}$ and found several examples of failed eruptions. After a preliminary inspection, three of them were classified as eruptions stopped by overlaying magnetic field structures. The events investigated are summarized in Table 1.

In the EUV range all events were observed mainly with the $171 \AA$ filter. The TRACE data were reduced with the standard trace_prep procedure available in the SolarSoftWare library. Even after reduction the TRACE images pointing is usually incorrect by some arcsec. Therefore the pointing should be corrected before the data are compared with those obtained with other instruments. We corrected the TRACE pointing according to the method described by T. Metcalf ${ }^{2}$. We used SOHO/EIT images obtained with the $171 \AA$ filter as reference data and calculated the shift of TRACE images. The corrections were not higher than 14" in both directions. However, the correction is significant and important since the observed brightenings have small areas.

We searched for brightenings with a semi-automated method. They were determined based on a light curve for every pixel on a set of spatially correlated EUV images for the

\footnotetext{
1 http://trace.lmsal . com/Public/Gallery/Movies/

2 http://www . cora.nwra.com/ metcalf/TRACE/pointing. html
} 


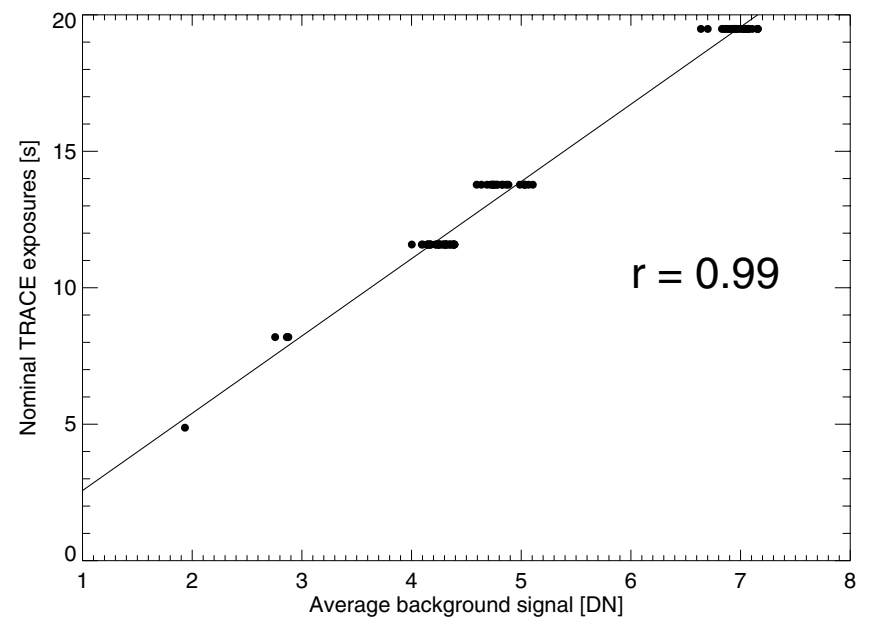

Fig. 1. Relationship between TRACE exposure time and average background signal level in images. The average background signal level was determined from the lower left image corner where the signal was dark, flat, and did not change substantially during the observation time interval. The correlation coefficient is shown in the plot.

whole event. The main requirement for the pixel to be classified as a brightening was a sudden increase in signal. To reduce the number of false brightening pixels (such as caused by cosmic rays), we also required that the increased signal appeared for at least two subsequent images and was surrounded by other brightened pixels. The group of neighboring pixels constitutes an area of brightening. The cumulative light curves presented below are calculated based only on signals from pixels that were marked as an area of brightening.

A similar method for finding brightened pixels was used by Mrozek \& Tomczak (2004). The method was effective in searching for impulsive soft X-ray (SXR) brightenings. However, in TRACE images we can expect several features that might be recognized as brightenings, but they are not and have to be manually excluded. For this reason we had to decide in each case which of the brightenings was real. The main problems were connected with solar energetic particles, moving structures, and a diffraction pattern. Solar energetic particles cause brightenings that can be classified by automatic methods. Accordingly, we filtered the maps of brightened pixels and removed structures smaller than four squared pixels. Solar energetic particles can also produce lines on images, therefore we also excluded brightened structures with a width equal to one to two pixels. Our method of finding brightened pixels has a tendency to find moving structures if they occur. These structures were removed manually from the map of brightenings.

Inspection of the TRACE reduced image cube used for the SOL1999-10-22T09:16 event showed that some corrections for the exposure times were also necessary. Namely, abrupt jumps of unknown origin in the light curve rates for all pixels were detected. The jumps in rate value were present even in dark featureless portions of the images where only background was observed. We checked the information on image exposure times stored in TRACE FITS file headers and found that these jumps occur when substantial changes in exposure time of the images were made. On the other hand, we observed that there the linear relationship between the average background signal level and TRACE exposure times for many of the images from other analyzed events is very good (see Fig. 1). This relationship also allows one to determine the exposures directly from the background signal. We compared the exposures taken from TRACE data files with those calculated from the background and found large discrepancies between them for some images. For these images we used calculated exposures. This allowed us to eliminate the jump effects in light curve rates.

The last feature that produces artificial brightenings is a diffraction pattern that is always present in TRACE images (Lin et al. 2001). It is a particularly severe problem in the case of very bright areas. The diffraction cross consists of almost exact, but fainter copies of a central source with decreasing brightness. The details of the diffraction cross and TRACE $171 \AA$ point spread function (PSF) are given in Gburek et al. (2006). If the intensity of a bright source changes, the diffraction sources also change intensity and are classified as brightened areas. We examined all brightened areas and checked whether they are real or are the result of the diffraction. An example is presented in Fig. 4 (panels from 05:23:09 UT to 05:26:50 UT), showing the situation we observed for SOL2004-07-14T05:23 event. The brightened area was observed, but it was masked by diffraction sources. Therefore we excluded this event from further analysis.

The next step in our analysis was the estimation of the eruption front that is measured above a reference point. In each of the three events we observed a very well defined area where the eruption was rooted. This we defined as the reference point as the base of the eruption. The analyzed expanding structures have a well-defined leading front, therefore the errors in the height estimate are relatively low. We calculated the uncertainties of the leading front position by measuring the position of the front in several different locations. Next we analyzed the spread of the values and found the maximal error to be equal 4 pixels $\left(2^{\prime \prime}\right)$. The estimated heights of the leading front of the eruption were fitted with cubic splines. The velocity was obtained through differentiating the fits. The differentiation of noisy data may lead to substantial error magnification. The problem of differentiating noisy data is widely discussed in the literature (Kontar \& MacKinnon 2005; Wen et al. 2007; Temmer et al. 2010). However, we were interested mainly in estimating approximate time intervals of the decreasing velocity. Consequently, the problem of error magnification is not as severe because we did not analyze individual minima or maxima in velocity and acceleration.

The absolute height of the eruption was difficult to obtain since we have only 2D information about 3D distribution of magnetic structures. We chose events located close to the solar limb to minimize the projection effects. Moreover, we can calculate the projection of real structures on the sky plane. Thus, we were able to obtain more realistic heights of erupting structures. The description of the method is given in the appendix.

\section{Data analysis}

\subsection{SOL1999-10-22TO9:16}

Figure 2 shows the eruption of a filament at solar limb, which took place on October 19, 1999. The associated flare was classified as C4.8 according to GOES. For this event there are available observations from TRACE at $171 \AA$ and YOHKOH/HXT observations for HXR. SOHO/LASCO recorded a CME that took place at the same time, which was observed on the east limb. It was not correlated to the analyzed flare since it was visible close to the west limb.

The whole evolution of this event is presented in Fig. 2. There are no TRACE images of the very beginning of the filament rise (between 9:08 UT and 9:11 UT) available. According to GOES, the flare began at 9:10 UT, and the maximal SXR flux was recorded at 9:16 UT. At that moment we also observed 


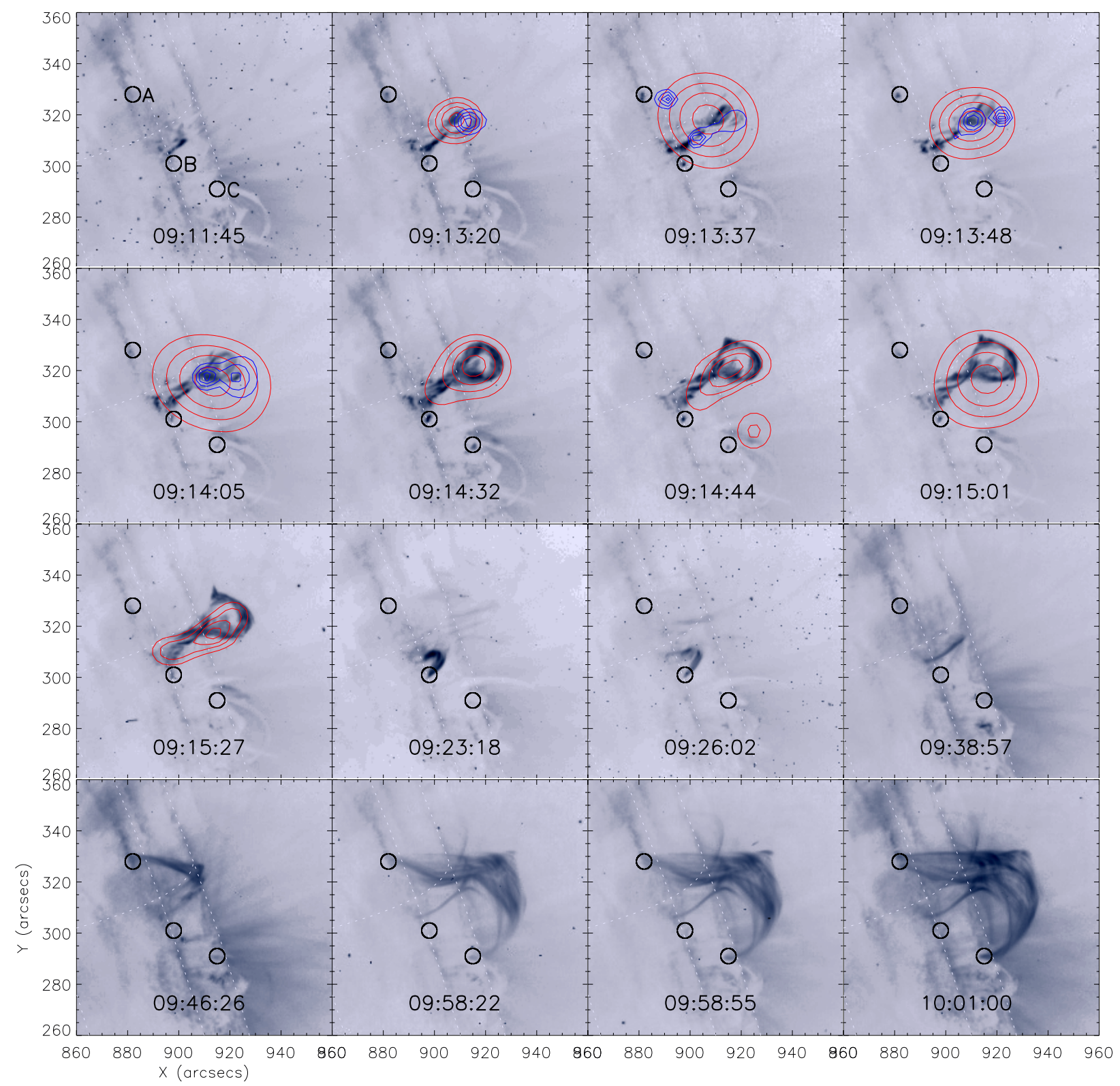

Fig. 2. Set of $171 \AA$ Amages taken from TRACE (171 ̊) for different moments during the evolution of SOL1999-10-22T09:16 event. Locations of brightenings are circled in every image. In the top-left image all regions are marked by letters. In selected images reconstructed HXR contours are marked for HXT L (dashed line) and M1 (solid line) for 30, 50, 70, and 90\% of maximum brightness. See the electronic form for a color version of the plot and for a movie presenting the entire event.

the maximal height of the analyzed eruption (Fig. 3, top panel). During the rising phase the eruption had its greatest velocity around 9:13 UT and at around 9:14 UT it was slowed down. The system of EUV post-flare loops was observed starting from 9:20 UT. They reached a maximum height equal to $8 \times 10^{3} \mathrm{~km}$. Moreover, at around 9:40 UT we observed the abrupt occurrence of high loops (their height is about $2.8 \times 10^{4} \mathrm{~km}$ ) that are a separate system, not related to the post-flare loops. They are high and can be compared to the maximal height of the eruption front.

We found three regions that meet the criterion for a chromospheric brightening. They are marked as A, B, and C in the top-left panel of Fig. 2. The EUV light curves for those regions are shown in Fig. 3 (panels d-f). Their shape resembles the shape of the velocity curve as well as that of the HXR curve. There are two maxima at 9:13 UT and 9:14 UT observed in the EUV light curves. The first maximum correlates well with the maximum in
HXR light curve but the second one happened about $15 \mathrm{~s}$ after the corresponding HXR maximum. The maxima on the light curves are observed simultaneously with a slowing down of the eruption. In all regions the first maximum precedes the moment of the slow-down of an eruption. The two brightening regions A and $\mathrm{C}$ are located near the footpoints of the high system of loops (Fig. 2, panel at 10:01 UT), and region B is located close to the footpoint of the flaring structure (Fig. 2, panel at 09:26 UT).

The HXR sources were reconstructed for two HXT channels, L and M1. We chose time intervals that cover the times of TRACE images. The L-channel source is large and diffuse and is spatially correlated with the evolving eruption. The HXT/M1 channel sources are significantly smaller and their behavior is much more interesting. Namely, the first source is visible several seconds before the maximum burst of HXR radiation (Fig. 2) and is located on the top of the eruption front. In the second 

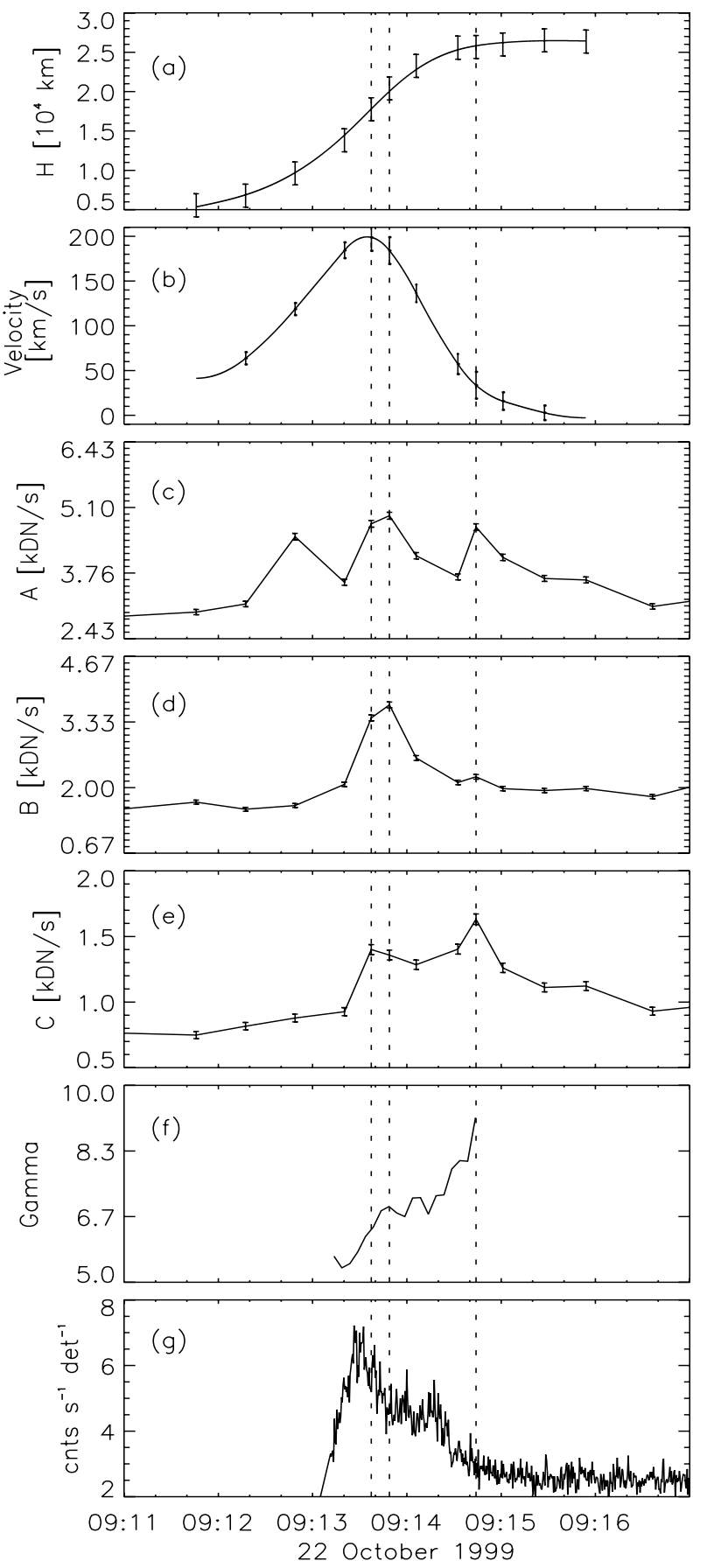

Fig. 3. SOL1999-10-22T09:16. Panels a) and b) show height and velocity against time. The height measurements were fitted with cubic splines. The velocity is a derivative of the obtained fit. Panels c)-e) present light curves for the brightening regions $\mathrm{A}, \mathrm{B}$, and $\mathrm{C}$. Panel f) shows gamma against time. Panel g) shows the HXR light curve obtained from the HXT instrument onboard YOHKOH in the M1 channel. The vertical dashed lines represent maxima of light curves for brightening regions in all panels.

image we observe two weak, compact sources located lower in the corona. They are spatially correlated with the EUV brightenings A and B. The correlation between the HXT/M1 source and the brightening A (the footpoint of the large magnetic structure observed later) may be the result of a nonthermal electron beam. Moreover, there is a weak HXT/L emission source that correlates with the area C (Fig. 2) observed at 9:14:44 UT - the moment of the strongest brightness observed in $\mathrm{C}$. These high loops are invisible at the time of eruption, but can be traced in images taken 30 min later. This suggests that we observe the electrons accelerated during the interaction between the eruption front and the system of loops located above. The next image (Fig. 2, top-right panel) shows HXT/M1 sources located again within the eruption. One of them is similar to sources observed by Liu \& Alexander (2009), who reported HXR sources in the $\mathrm{X}$-point of the eruption. The second source is located at the front of the eruption and is possibly another manifestation of the reconnection between the eruption and the overlaying high loops. We found a similar situation (X-point source and at-the-front source) for the next moment (09:14:05 UT), but the sources are weaker and more diffuse due to the low level of measured signal. The observed sources occurred during the strongest HXR burst, during the deceleration of the eruption, and they are correlated with the occurrence of EUV brightenings.

\subsection{SOL2004-07-14T05:23}

The second of the failed eruptions was observed on July 14, 2004 and was accompanied by an M6.2 flare. The event was observed in the active region NOAA 10646 . This event was also analyzed in Mrozek (2011) as an example of a failed eruption that started vertical oscillations of the overlaying coronal loops.

For this event observations from TRACE (171 $\AA$ ) and RHESSI are available. The event SOL2004-07-14T05:23 had no associated CME. According to GOES, the flare started at 5:02 UT and reached the maximum brightness at 5:23 UT. The evolution of the entire event is presented in Fig. 4. It can be divided into two phases. The first, between 5:17 UT and 5:24 UT, when the eruption rose up to its maximum height (Fig. 5). During this phase the highest HXR signal was recorded at the beginning of the eruption rise. The maximum in HXR is correlated with the lowest value of HXR's photon spectral index and is observed close to the maximum of the velocity. The end of this phase took place at 5:24 UT when the rising eruption stopped. At that time the main front of the eruption is held at almost the same height. The next phase started at 5:27 UT when the abrupt change of the main front direction appeared (Fig. 4, at 5:28.40 UT). We treated it as a side eruption front and therefore did not include it in the height analysis. At that moment also small post-flare loops became apparent at the location where the eruption started to rise (Fig. 4, panels for 5:35 UT and 5:50 UT).

From 6:57 UT a system of high loops started to be visible. Again, as for the SOL1999-10-22T09:16 event, their height $\left(5.5 \times 10^{4} \mathrm{~km}\right)$ corresponds to the height at which the eruption was confined in the first phase. The high-lying system of loops is a structure distinct from the small post-flare arcade (Fig. 4, panels from 5:26:50 UT to 5:50:34 UT). The small post-flare loops reach a maximum height equal to $10^{4} \mathrm{~km}$. Moreover, the footpoints of the post flare arcade and the footpoints of the high loops are located in different locations.

There are two regions classified as brightenings (Fig. 4, topleft panel), but one of them overlaps with diffraction pattern on TRACE images. Thus, its emission was affected by the changes of brightness of the footpoints of the flare. Accordingly, this brightening was excluded from the subsequent analysis. The light curve for the remaining region has three distinct local maxima (Fig. 5). All of them occurred when the systematical decrease of velocity of the eruption was observed. They correlate with local drops in the HXR photon spectral index. The third maximum took place at almost the same moment as the beginning of the side eruption stated. Region A is located far away 


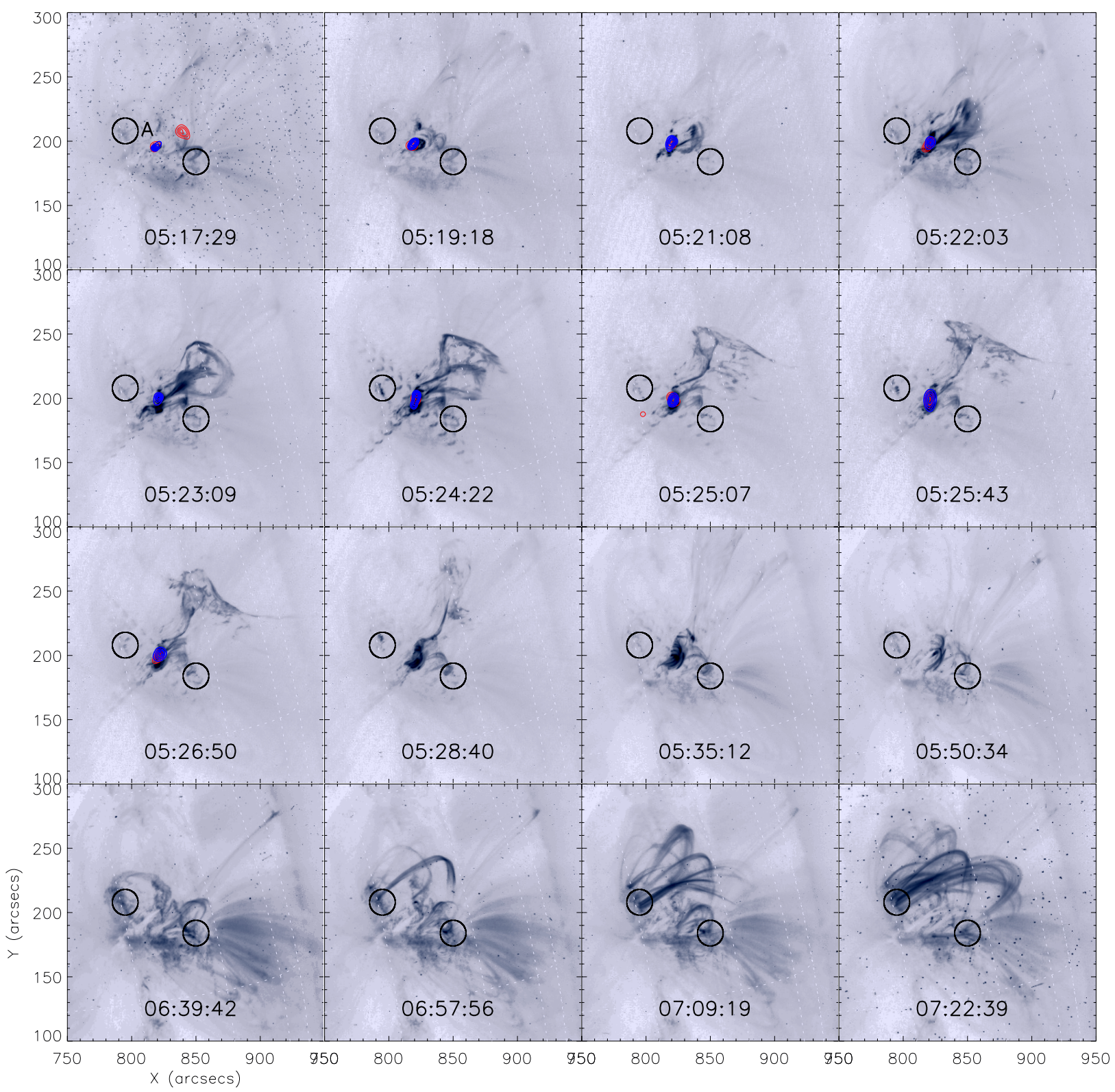

Fig. 4. Set of $171 \AA \AA$ images taken from TRACE for different moments during the evolution of the SOL2004-07-14T05:23 event. The location of the brightening is circled in every image. In the top-left image the region is named. In selected images reconstructed HXR contours are marked for the RHESSI energy bands at 6-12 keV (white solid line) and at 25-50 keV (black solid line). See the electronic form for a color version of the plot and for a movie presenting the entire event.

from the place where the eruption started to rise. However, it is located at one of the footpoints of the high loop system (Fig. 4, panel for 7:09 UT).

The entire event was observed by RHESSI, which allowed us to reconstruct images and perform fits to the observed spectra. We reconstructed HXR images in two energy channels, 6-8 keV and $14-25 \mathrm{keV}$. The HXR emission sources are very compact and are spatially correlated with the flare site. We did not record any significant emission located at the front of the erupting structure. However, in a few reconstructed images we observed a very weak HXR source located close to region A. The emission was below $10 \%$ of the maximum, hence it is not presented in Fig. 4.

The HXR light curves and the HXR photon spectral index evolution show some interesting coincidences with EUV brightenings and kinematics of the eruption. Namely, we did not observe any significant brightenings of area A during the impulsive phase, which suggests that there is no connection between the flare and the region $\mathrm{A}$. We detected significant changes of brightness of this region during the braking of the eruption front (Fig. 5). The strong EUV brightenings were accompanied by the very weak HXR brightenings (12-25 keV). There are also simultaneous episodes of the spectral hardening. Taking into account the overall behavior, we conclude that area A is a good example of an area that was hit by nonthermal electrons accelerated during the eruption braking due to interaction with overlaying field.

\subsection{SOL2004-08-13T18:12}

The last of the analyzed events happened on August 13, 2004 in the active region NOAA 10656. According to the GOES classification, it was an X1.0 flare whose maximum flux was observed 


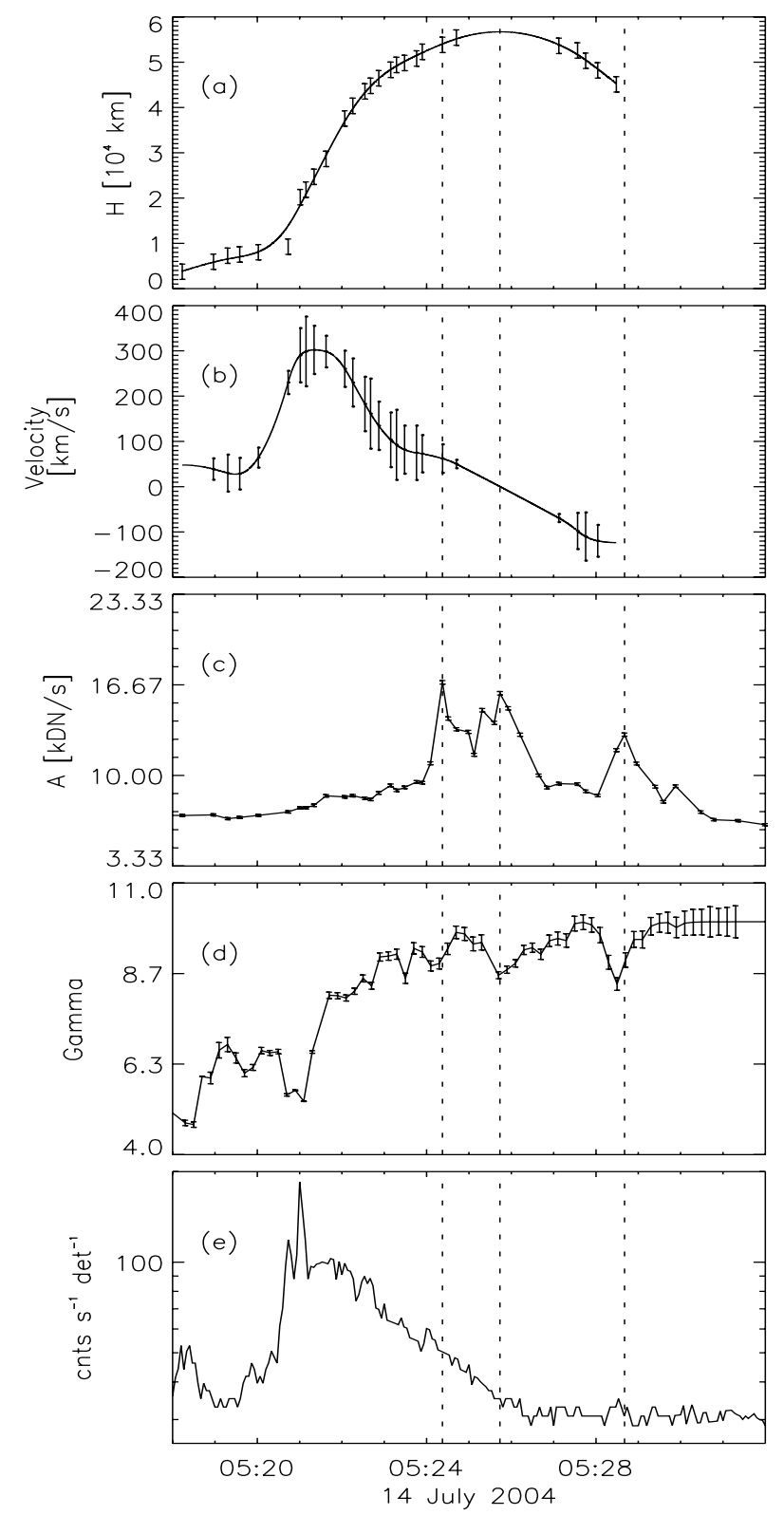

Fig. 5. SOL2004-07-14T05:23. Panels a) and b) show height and velocity against time. The height measurements were fitted with cubic splines. The velocity is a derivative of the obtained fit. Panel c) presents light curves for the brightening region A. Panel d) shows gamma against time. Panel e) shows the HXR light curve obtained from the RHESSI (12-25 keV). The vertical dashed lines represent maxima of light curves for brightening regions in all panels.

at 18:12 UT. There are TRACE (171 $\AA$ ) observations for the EUV range. The HXR emission was detected by RHESSI for the entire flare. The evolution of this event is illustrated in Fig. 6. A CME associated with this event was observed.

The flare began at 18:07 UT according to GOES. In contrast to the previous events, the high system of overlaying loops is visible even in images taken before the eruption start. The height of these loops is equal to $9.4 \times 10^{4} \mathrm{~km}$ before the beginning of the eruption. From 18:10 UT we observed the development of the eruption. Simultaneously, the overlaying loops were observed stretching outward, as can be seen in Fig. 7. The movement of the loops overlaying the eruption stopped at 18:21 UT since the eruption was fully stopped at 18:24 UT. After that typical post-flare loops became visible around the region where the eruption started. Their height is equal to $1.2 \times 10^{3} \mathrm{~km}$.

We observed a clear interaction between the eruption and the overlaying loops, which is presented in Fig. 7. We analyzed the changes of the overlaying loop heights along a cut that is presented in the left panel of Fig. 7. The overlaying loops were observed to rise together with the evolving eruption. Obviously, we have only a 2D image and there is no exact information about the direction of this movement. It is possible that we observed an eruption that elbows overlaying structures and there is no stretching. If the overlaying loops were pushed to a side, we should observe an initial lowering of the loop tops because of the projection. We did not observe such a behavior (Fig. 7, right panel) and treated it as a suggestion for rising height.

We found five regions that met the chromospheric brightening criteria. They are labeled from A to E (Fig. 6, top-left panel). Their light curves are presented in Fig. 8. Regions A to D are located near one of the footpoints of the overlaying high loops. Only region $\mathrm{E}$ is located close to the second footpoint, but it is probably a footpoint of another system of loops. The reconstructed HXR contours (6-8 keV and 25-30 keV) are spatially correlated with the flaring structure. We tried to reconstruct HXR sources with different time intervals and energy ranges but found no significant emission correlated with either the EUV brightenings or the eruption.

Each of the regions brightened at a different moment in time, but all of them slightly precede the moment at which the overlaying magnetic loop ceased to move. The light curve for region $\mathrm{C}$ shows an increase in signal at the moment of the maximum in the HXR curve (Fig. 8). The intensity of this region initially rises and stays almost at the same level between 18:11 UT and 18:13 UT. After this time the intensity slowly decreases to the background level. The light curve of region A has a similar shape, but its maximum is closer to the second-highest HXR peak at around 18:13 UT. The light curve of region D has two distinctive maxima, the first one of which is well-correlated with the minimum of the HXR photon spectral index. The light curve of region $B$ has its maximum near the highest HXR maximum. The light curve of region $\mathrm{E}$ shows weak reaction correlated with the HXR peak around 18:15:30 UT. All of them happened during the beginning phase of the eruption. The last brightening was observed around 18:13 UT. After that time we observed the next phase of velocity rising. This behavior strongly supports the scenario that assumes that the brightenings occur during episodes of interaction between the eruption and the overlaying large system of loops.

\section{Results}

\subsection{Time correlations}

We searched for correlations between the kinematics of the eruption, EUV brightenings, HXR emission, and the HXR photon spectral index. In all three cases we found that such a relation exists. Usually, we observed the HXR burst first and shortly after that we detected the local minimum of the HXR photon spectral index. Simultaneously, we observed a decrease in velocity. These features were usually observed just before or simultaneously with EUV brightenings. The EUV brightenings were never observed before the HXR burst and they were correlated to the decrease of the eruption velocity. The time shift observed was usually less than several seconds.

In the case of SOL2004-07-14T05:23 we observed a series of EUV brightenings that occurred during the long (about 6 min) 


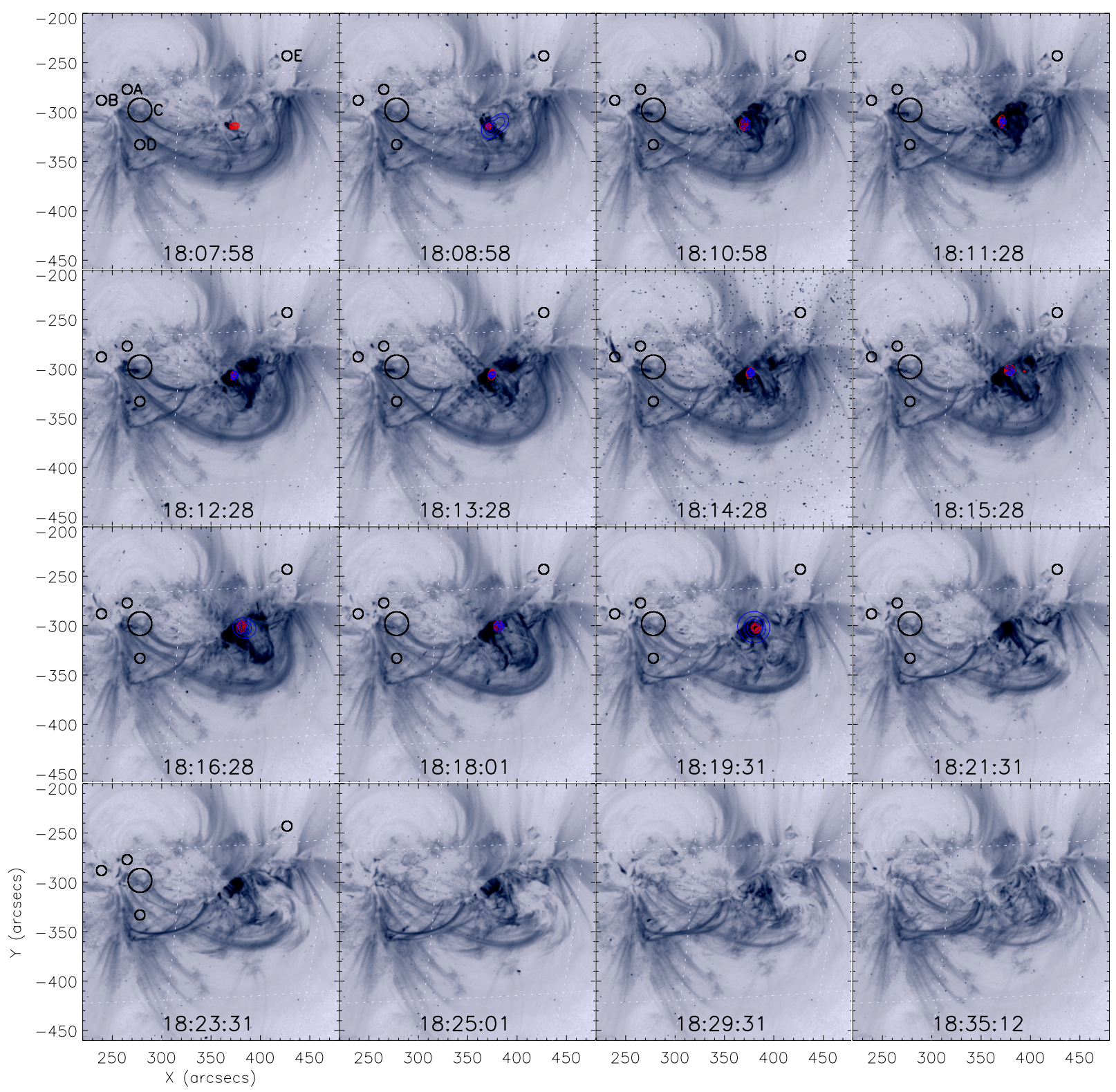

Fig. 6. Set of $171 \AA$ A images taken from TRACE for different moments during the evolution of the SOL2004-08-13T18:12 event. The locations of the brightenings are circled in every image. In the top-left image the regions are named. In selected images reconstructed HXR contours are marked for the RHESSI energy bands at 6-12 keV (white solid line) and at 25-50 keV (gray solid line). See the electronic form for a color version of the plot and for a movie presenting the entire event.
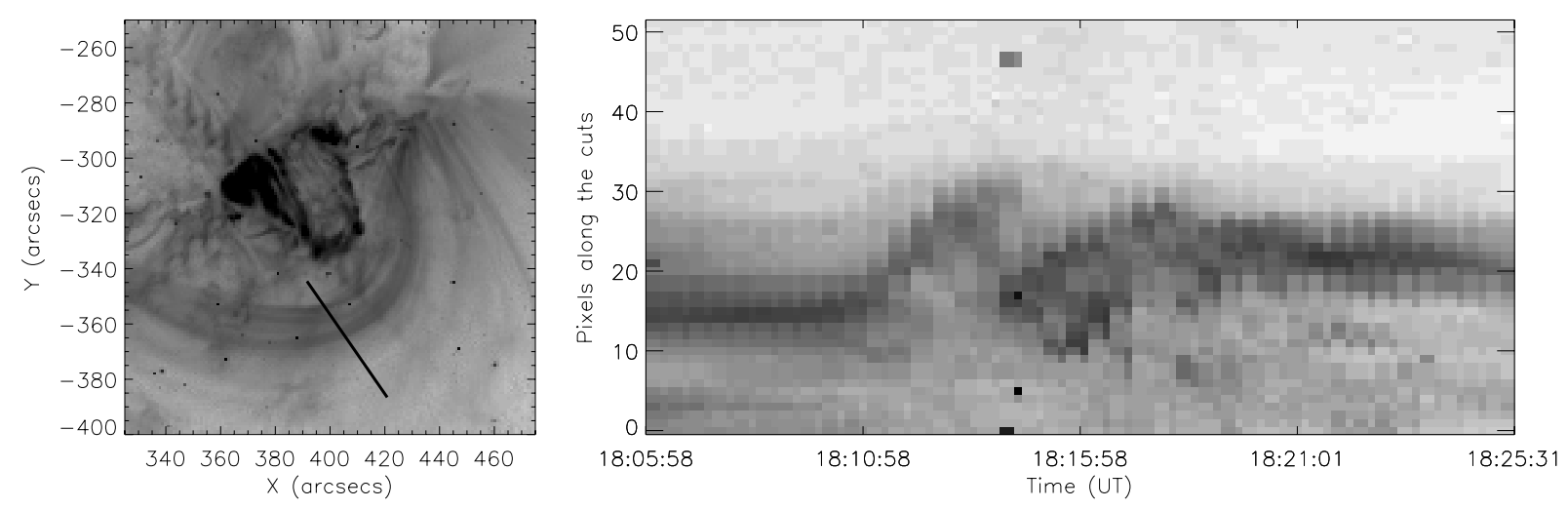

Fig. 7. Example of the cut through the overlaying system of loops for SOL2004-08-13T18:12. 

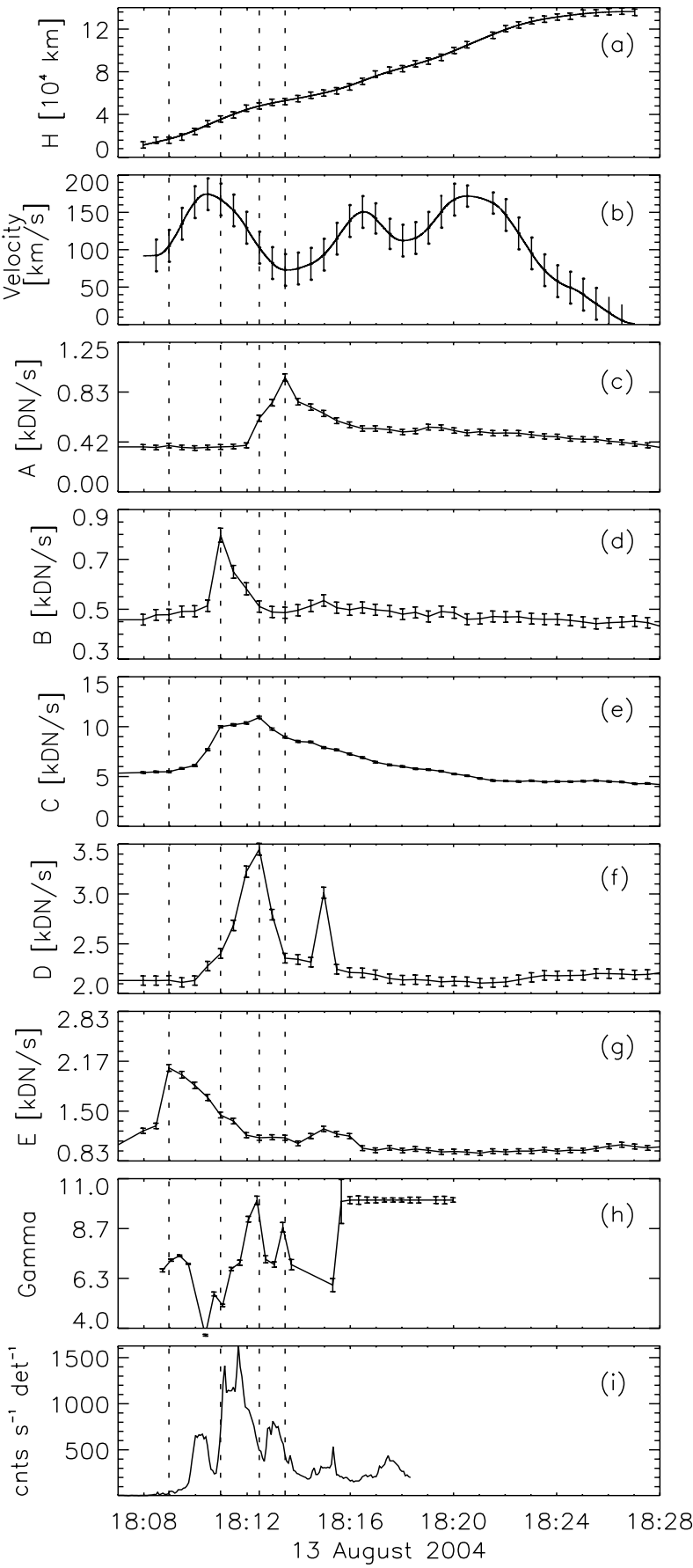

Fig. 8. SOL2004-08-13T18:12. Panels a) and b) show height and velocity against time. The height measurements were fitted with cubic splines. The velocity is a derivative of the obtained fit. Panels c)-g) present light curves for the brightening regions A, B, C, D, and E. Panel h) shows gamma against time. Panel i) shows the HXR light curve obtained from the RHESSI (25-50 keV). The vertical dashed lines represent maxima of light curves for brightening regions in all panels.

stage of the eruption braking. These brightenings were observed when HXR emission (12-25 keV) was on the decay phase. However, we measured a significant hardening of the HXR spectra, which is well correlated in time with EUV brightenings. Consequently, we assume that these brightenings are caused by nonthermal electron beams.

In the SOL2004-08-13T18:12 event we observed overlaying loops since the early phase of the eruption evolution. The
EUV brightenings were observed only during the initial phase of the eruption. It was the phase when the overlaying loops were observed to change their height as well (Fig. 7). At almost the same time at which the overlaying loops reached their maximum height, we observed the last EUV brightening in the footpoint of these loops. After that we still measured an increase of the eruption. It is possible that the eruption pushed through the overlaying field and the interaction with overlaying field ended. The observational signature for this scenario is the associated CME - clearly visible, but very slow and only a few degrees wide.

\subsection{Spatial distribution of the brightenings}

In all events we found EUV brightenings that were observed during the evolution of the failed eruptions. The majority of these brightenings was spatially correlated with extended magnetic structures that enclose the eruption. These large systems of loops (height up to $6 \times 10^{4} \mathrm{~km}$ ) are not typical post-flare loops. We observed a system of post-flare loops that evolved with continuously rising height but in the analyzed events their maximum height was less than $10^{4} \mathrm{~km}$. Analyzing the occurrence of post-flare loops, we found a discontinuity in heights between low-lying and high-lying loops (see the movies in the online material). It suggests that there is a weak relationship between them. Moreover, the EUV brightenings observed are located at the footpoints of these large loops. The EUV brightenings are not flare footpoints and are observed far away from the flare site. The typical distance between the flare site and the EUV brightenings exceeds $2 \times 10^{4} \mathrm{~km}$.

The large system of overlaying loops is closely related with the maximal height reached by the eruption. The observations suggest that there is an interaction between the eruption and the overlaying structures. For example in the SOL2004-0714T05:23 event we observed the change of the front shape at heights similar to those of the large loops visible an hour after the flare. In the case of SOL1999-10-22T09:16 we observed the 23-33 keV emission sources at the top of the eruption, which suggests some episodes of energy release. In the SOL2004-0813T18:12 we observed the overlaying system of loops that were visible during the overall evolution of the eruption and the overlaying loops changed height according to the eruption.

We found a correlation between the HXR emission source and the EUV brightening in one case only (SOL1999-1022T09:16). However, some of reconstructed HXR images suggest that such sources may exist, but they are very weak and are strongly masked by flare emission.

\subsection{Estimation of $H X R$ source brightness}

Temporal characteristics of the brightenings show that the time shift between an HXR peak and the maximum of EUV brightenings is very short. This suggests that we may exclude mechanisms such as waves or mass draining, which operate on different time scales. Thus, we have to answer the question: why do we not see spatially correlated HXR sources if the EUV brightenings originate in the nonthermal electrons beam?

To answer this question we recall the analysis of Mrozek et al. (2007), where EUV and UV brightenings observed in flare footpoints were compared with HXR emission sources. These authors selected a group of flares with well-developed impulsive phases and clearly observed footpoint sources. The analysis was based on TRACE and YOHKOH/HXT data. TRACE footpoints are usually saturated during the impulsive phase. The authors 


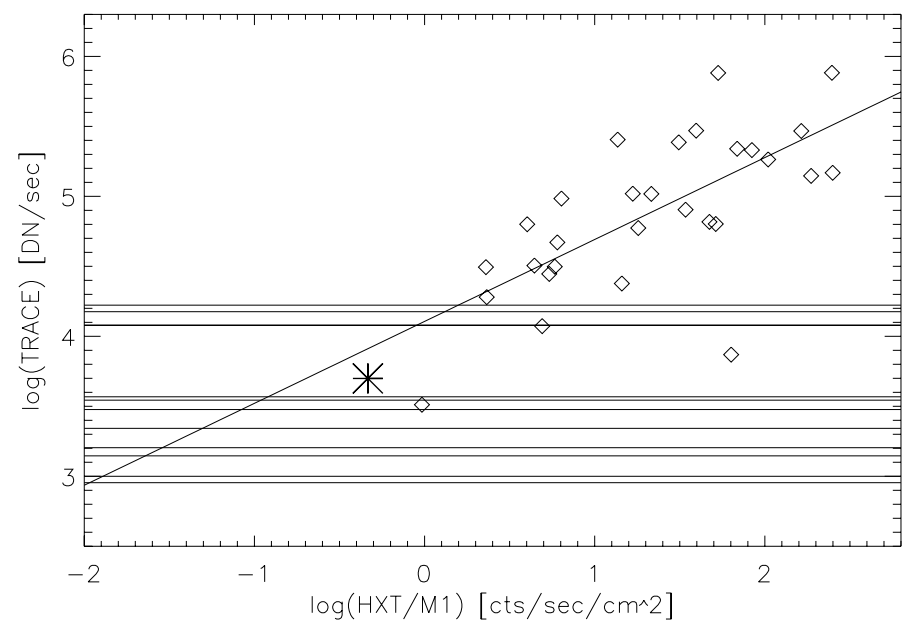

Fig. 9. Correlation between the TRACE $171 \AA$ and HXT/M1 signals. The results obtained by Mrozek et al. (2007) are presented with diamonds. The linear fit to these points is also presented. One point estimated for source A from the SOL1999-10-22T09:16 event is presented with an asterisk. The horizontal lines present the brightness levels obtained for the other EUV brightenings.

developed a method for saturated signal recovery based on the TRACE diffraction pattern characteristics (Gburek et al. 2006). The EUV signal recovery allowed a quantitative analysis of EUV and HXR signals. The results obtained showed that the signals were correlated, and we conclude that both types of sources were caused by nonthermal electrons. The correlations between the EUV (171 ̊) and the HXR (HXT/M1 channel) fluxes are presented in Fig. 9.

Tomczak (1999) analyzed SXR brightenings in flare footpoints and compared them to HXR emission sources. He concluded that the observed correlations may be used for analysis of nonthermal electron stream if no HXR sources are observed. According to this, we decided to use the correlations obtained by Mrozek et al. (2007) to estimate the expected intensities of HXR sources that are spatially correlated with the observed EUV brightenings. For the events analyzed here we found that none of brightenings was saturated, therefore we did not need to perform any signal recovery. The EUV intensities of brightenings analyzed in this paper are marked as horizontal lines in Fig. 9. One point representing the brightening from SOL199910-22T09:16 event is presented with an asterisk. All horizontal lines cross with dependence obtained for flare footpoints in the left side of the panel. This means that any hypothetical HXR sources that may be spatially correlated with the analyzed brightenings are very weak. They are at least ten times fainter than the faintest footpoint sources observed with the HXT. However, the main problem is not the intensity of the sources, but rather the dynamic range. In Fig. 9 there is a group of horizontal lines located at a level that may enable a HXR source detection. Unfortunately, at that time the HXR images were dominated by bright sources connected with a flaring structure.

\subsection{Heating and cooling the high-lying system of loops}

Footpoints of a large system of loops observed in the EUV are dim and are even undetectable in HXR. This means that there are few electrons accelerated during the interaction between an eruption and higher loops. Thus the following question may occur: Is the energy deposited in footpoints sufficiently high to evaporate chromospheric material, i.e., heat the material to $1 \mathrm{MK}$ and transport it high into the corona?

We can answer the question with a straightforward estimation for event No. 1. The large system of loops is observed in the TRACE $171 \AA$ band, i.e., the plasma should have a temperature of about $1 \mathrm{MK}$. The height and volume of the loops can be estimated also from the TRACE images. The density of a typical EUV loop is about $10^{9} \mathrm{~cm}^{-3}$ (Aschwanden et al. 1999). Based on these parameters, we calculated that the total thermal and gravitational energy of the plasma contained in the loops is about $5 \times 10^{26}$ erg.

On the other hand, energy deposited by nonthermal electrons in the HXR footpoint of event No. 1 can be calculated based on a reconstructed HXT/M1 source and correlations between HXR and EUV signals and the power-law index of the HXR spectrum obtained by Mrozek et al. (2007). We estimated that the energy is about $10^{28} \mathrm{erg}$. This is more than the total thermal and gravitational energy contained in the large EUV loops. The energy deposited by nonthermal electrons is sufficiently high to heat chromospheric material to 6-7 MK and to raise it to fill the whole large system of loops with plasma with a density of about $5 \times 10^{9} \mathrm{~cm}^{-3}$. Loops filled with such plasma should be visible in SXR.

The observations for the SOL1999-10-22T09:16 event confirm this expectation. We carefully inspected $\mathrm{YOHKOH/SXT}$ data and found a few SXT/A1.1 (maximum of thermal sensitivity $5 \mathrm{MK}$ ) images that partly overlap with the analyzed TRACE images. Namely, the southern leg of the high-lying loop is visible close to the edge of the SXT/Al.1 frame. The temperature response of the SXT/Al.1 filter has a maximum close to $5 \mathrm{MK}$, which is consistent with the estimate made above. Part of the leg is visible between 9:25 UT and 9:43 UT in the SXT/Al.1 images. In the full-Sun image taken with the AlMg (maximum of thermal sensitivity $6 \mathrm{MK}$ ) filter on 9:04:55 UT there is no such structure.

If we assume that heating the loops to 5-7 MK through interaction may occur, another problem may arise. Namely, the time difference between the eruption stop and the occurrence of highlying loops is about 40-90 min. If we interpret these loops as a system that was heated by the interaction with the eruption and cooled down later, the loops should be visible much earlier, assuming typical values of cooling times (Antiochos \& Sturrock 1978).

However, recent observations (Winebarger et al. 2003) show that we must be careful when analyzing the visibility of EUV loops and relating them to hydrostatic cooling times. Winebarger et al. (2003) analyzed five loops observed by TRACE with EUV filters. These authors estimated the time of the appearance of a loop in a given filter. The estimated difference of the appearance in TRACE $195 \AA$ and $171 \AA$ filters varies from $150 \mathrm{~s}$ to $11000 \mathrm{~s}$. In one case (loop 2 in Winebarger et al. 2003) observations made in three EUV filters were available. The time difference of loop visibility for the $284 \AA$ and $171 \AA$ filters was about $50 \mathrm{~min}$. The maxima of the temperature response of these filters are $2 \mathrm{MK}$ and 1.3 MK, respectively. This means that the loops in the $171 \AA$ filter may be visible even an hour after they are heated to temperatures of a few MK.

With the present set of data we are not able to resolve the temperature evolution of the high-lying loops with time. We found a few SXT/A1.1 images for the SOL1999-10-22T09:16 event. The images overlap with the southern part of our high loops, therefore not the whole structure can be observed. But, the southern leg is visible in the SXT/Al.1 images between 9:25 UT 
and 9:43 UT. Moreover, we inspected SOHO/EIT $195 \AA$ A images. The high-lying system of loops is visible starting from 9:48 UT, but we must keep in mind that the temporal resolution of EIT data is $12 \mathrm{~min}$.

The interaction between an eruption and overlaying magnetic structures is a complex process. We have to remember that the estimated energy contained in a nonthermal electron is only a part of the energy released during the interaction. Detailed numerical simulations are needed to explain the different interaction features.

\section{Discussion and conclusion}

We presented observations of three failed eruptions recorded by TRACE observed with the $171 \AA$ filter. The EUV observations are supplemented by HXR data from YOHKOH/HXT and RHESSI. We used a semi-automated method to search for regions that abruptly brightened in the TRACE images. Regions that were classified as EUV brightenings are usually located far away from flare loops, so they are not directly correlated with processes there.

We found some common behavior of brightenings. The temporal and spatial characteristics may be summarized as follows:

- The characteristics analyzed (height, velocity, HXR photon spectral index, EUV brightenings, HXR light curves) are time-correlated. Usually an HXR peak and the minimum of an HXR photon spectral index are observed a few seconds before an EUV brightening.

- The maximum EUV intensity of selected regions was observed during the deceleration of an eruption front.

- The EUV brightenings were found using a semi-automated method. They are in the exact same positions as the observed footpoints of the large systems of loops that are observed tens of minutes after the eruption braking episodes. These loops are not typical "post-flare loops".

- The heights of the large system of loops are almost the same as the maximum height reached by the eruption. Together with the fact that the brightenings are observed in the footpoints of these loops, this suggests that the loops exist since the beginning of the eruption, but for some reason (temperature, density) are not visible.

- The interaction with the eruption causes the heating of the loops and, after they are cooled down, the loops become visible in the EUV 30-90 min later. Warren et al. (2002) explained these long cooling times by assuming that loops have an unresolved filamentary structure. Multi-stranded loops can persist much longer than predicted with a characteristic cooling time. More hypotheses are possible (Winebarger et al. 2003), such as residual heating or nonionization equilibrium, but these require detailed hydrodynamical simulations.

- In one case we detected very weak HXR emission (23$33 \mathrm{keV}$ ) located exactly at the front of the eruption. This may suggest that some energy is released at the places of interaction between the eruption front and the overlaying magnetic field structures.

- Expected HXR sources connected with the EUV brightenings are weak and may rarely be observed with the present instruments. They are about ten times fainter than the faintest observed footpoints.

Based on the available data, we presented the following common scenario for the observed events (Fig. 10). This scenario should

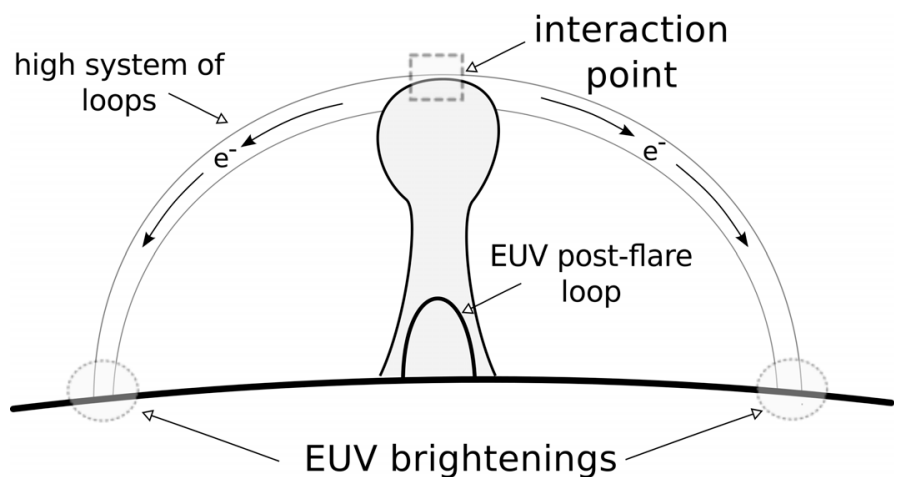

Fig. 10. Scenario suggested for the production of EUV brightenings.

not be confused with the so-called unified model (Shibata 1999) of a solar flare. The rising eruption, after the initial acceleration, is slowed down by the interaction with the overlaying magnetic field. Except for SOL2004-08-13T18:12 the overlaying field is not directly visible. In the points where the interaction between the erupting structure and the overlaying magnetic loop happens, particles are being accelerated to nonthermal velocities. Next, the particles propagate through probably almost empty magnetic loops (events Nos. 1 and 2) down to the transition region and the chromosphere where they collide with dense matter and produce EUV brightenings. If the chromospheric evaporation occurs, the loops are filled with plasma, and after cooling to $1 \mathrm{MK}$ they become visible in TRACE $171 \AA$ images.

The importance of the overlaying magnetic field in the confining eruptions was discussed in several theoretical and observational papers. The results presented here show that the interactions between an eruption and an overlaying field may be traced in the EUV and HXR ranges. In the follow-up paper we will present a quantitative analysis of these observations and will investigate the energy balance of the observed features.

Acknowledgements. The RHESSI and TRACE satellites are NASA Small Explorer (SMEX) missions. The YOHKOH satellite is a project of the Institute of Space and Astronautical Science of Japan. We acknowledge financial support from the Polish National Science Centre grants 2011/03/B/ST9/00104 and 2011/01/M/ST9/06096 and from the European Commission Seventh Framework Programme under the grant agreement No. 284461 (eHEROES project). We acknowledge many useful and inspiring discussions with Michał Tomczak. We also thank Barbara Cader-Sroka for editorial remarks. We are grateful to the anonymous referee for a fruitful discussion and many remarks that helped to improve the paper.

\section{Appendix A: Estimation of the absolute eruption height}

The height of an eruption above the photosphere is a very important parameter. It helps to understand kinematic evolution of this phenomenon. All heights estimated in this paper were corrected for the projection effect using the method described below. The method allows one to estimate the real altitude/height of any source/feature observed in the solar corona.

To estimate the real height of an eruption, we need to know its position in the TRACE image and find the point on the solar photosphere above which the eruption is situated (reference point). The eruption's position was defined by the position of its leading front. The position of the reference point was taken from the locations of footpoint sources visible simultaneously with the eruption in the TRACE images. 

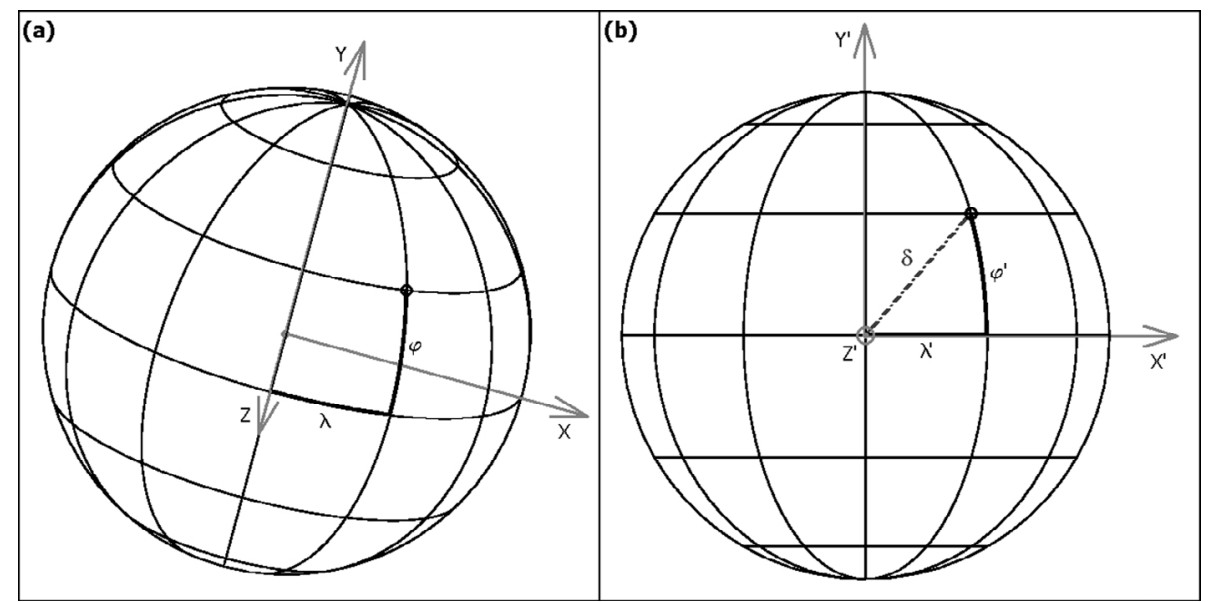

Fig. A.1. Heliographical and Cartesian coordinate systems used at the corrected altitude/height for the projection effect: a) real heliographical coordinate system $(\Lambda \Phi)$ and related Cartesian system $(X Y Z)$, b) rotated heliographical coordinate system $(\Lambda \Phi)^{\prime}$ and related Cartesian system $(X Y Z)^{\prime}$. The $z^{\prime}$-axis directed toward the observer.

For a single eruption we have several TRACE images in which the eruption was visible. The position of the leading front of the eruption was measured in each available image. The position of the reference point was taken from the one image in which the footpoint sources were the most pronounced. Because the time for which the position of the reference point was determined is somewhat different from the time of the leading front measurements, all positions were corrected to account for the solar rotation. For each pair of positions measured, leading front reference point, we are now able to estimate the corrected height of an eruption.

In the first step the apparent distance, $d_{\mathrm{a}}$, between the leading front position and the center of the Sun was calculated. To correct the apparent distance for the projection effect, we need to know the angle, $\delta$, between the direction to the observer and the local vertical direction passing through the reference point. If the solar rotation axis is perpendicular to the observer/Earth's orbit, the Sun and the heliographical coordinate system are visible to the observer as shown in Fig. A.1b. Then $\delta$ can be calculated directly from the heliographical coordinates of the reference point (longitude, $\lambda^{\prime}$ and latitude, $\phi^{\prime}$ )

$\cos \delta=\cos \lambda^{\prime} \cos \phi^{\prime}$

Unfortunately, the rotation axis of the Sun is inclined toward the observer/Earth's orbit at 82.75 degrees. Therefore the Sun and the heliographical coordinate system are visible at different inclinations at different points of the Earth's orbit, as shown e.g. in Fig. A.1a. At any time the inclination of the solar rotation axis as seen on the sky plane can be described by two angles: $\alpha-$ the inclination toward the eastern/western solar limb, and $\beta$ - the inclination toward the observer. For example the Sun is inclined with its north pole toward the observer and toward the western limb in Fig. A.1a. An accurate correction for the projection effect demands including $\alpha$ and $\beta$ in calculating the $\delta$ angle. The easiest way to do this is to convert the real coordinates of the reference point $(\lambda, \phi)$ to a new idealized heliographical coordinate system, shown in Fig. A.1b. For this purpose we define two heliographical coordinate systems and two related Cartesian coordinate systems, the centers of which are at the center of the Sun.

- The real heliographical coordinate system $(\Lambda \Phi)$ and the related system $(X Y Z)$. The $z$-axis of the system is crossing the solar photosphere in the point $(\lambda=0, \phi=0)$, the $x$-axis in the point $(0,90 \mathrm{~W})$ and the $y$-axis agrees with the solar rotation axis (see Fig. A.1a).
- A rotated (idealized) heliographical coordinate system $(\Lambda \Phi)^{\prime}$, in which the point $\left(\lambda^{\prime}=0, \phi^{\prime}=0\right)$ is situated at the center of the solar disk. The related system $(X Y Z)^{\prime}$ has the $z^{\prime}$-axis directed towards the Earth/observer. The $x^{\prime}$-axis and the $y^{\prime}$-axis lay in the sky/image plane. Thus, these axes constitute a heliocentric coordinate system that is used to measure positions of any point in, e.g., a TRACE image (see Fig. A.1b).

To change from system $(\Lambda \Phi)$ to system $(\Lambda \Phi)^{\prime}$, we have to rotate the first one by the angle $\beta$ about the $x$-axis to align the $z$-axis with the $z^{\prime}$-axis, then rotate by the angle $\alpha$ about the $z$-axis to align the $x$-axis and $y$-axis with the $x^{\prime}$-axis and $y^{\prime}$-axis, respectively. Equations that allow one to convert the Cartesian coordinates of a point from system $(\Lambda \Phi)$ to system $(\Lambda \Phi)^{\prime}$ are

$$
\begin{aligned}
& x^{\prime}=(z \sin \beta+y \cos \beta) \sin \alpha+x \cos \alpha, \\
& y^{\prime}=(z \sin \beta+y \cos \beta) \cos \alpha+x \sin \alpha, \\
& z^{\prime}=z \cos \beta-y \sin \beta,
\end{aligned}
$$

where $(x, y, z)$ are coordinates of a point in system $(X Y Z)$ and $\left(x^{\prime}, y^{\prime}, z^{\prime}\right)$-in system $(X Y Z)^{\prime}$. Since the coordinates of points on the 'surface' of the Sun are given in the heliographical coordinate system, we must convert Eq. (A.2) into this system. Using equations

$$
\begin{aligned}
& x=R_{\odot} \cos \phi \sin \lambda, \\
& y=R_{\odot} \sin \phi, \\
& z=R_{\odot} \cos \phi \cos \lambda,
\end{aligned}
$$

$$
\begin{aligned}
& x^{\prime}=R_{\odot} \cos \phi^{\prime} \sin \lambda^{\prime}, \\
& y^{\prime}=R_{\odot} \sin \phi^{\prime}, \\
& z^{\prime}=R_{\odot} \cos \phi^{\prime} \cos \lambda^{\prime},
\end{aligned}
$$

where $R_{\odot}$ is the Sun's radius, $(\lambda, \phi)$ and $\left(\lambda^{\prime}, \phi^{\prime}\right)$ are coordinates in the systems $(\Lambda, \Phi)$ and $(\Lambda, \Phi)^{\prime}$, respectively, after simple transformations we obtain

$$
\begin{aligned}
\tan \lambda^{\prime} & =\frac{a_{1} \sin \alpha+a_{2} \sin \alpha+a_{3} \cos \alpha}{\cos \phi \cos \lambda \cos \beta-\sin \phi \sin \beta}, \\
\sin \phi^{\prime} & =a_{1} \cos \alpha+a_{2} \cos \alpha+a_{3} \sin \alpha,
\end{aligned}
$$

where

$$
\begin{aligned}
& a_{1}=\cos \phi \cos \lambda \sin \beta, \\
& a_{2}=\sin \phi \cos \beta, \\
& a_{3}=\cos \phi \sin \lambda .
\end{aligned}
$$

The angles $\alpha$ and $\beta$ for any date/time can be estimated using the SolarSoftWare procedures.

Using Eq. (A.4) we can convert the measured $(\lambda, \phi)$ coordinates of the reference point into the much more suitable $\left(\lambda^{\prime}, \phi^{\prime}\right)$ 
coordinates and then calculate the angle $\delta$ from Eq. (A.1). Now, the apparent distance, $d_{\mathrm{a}}$, between the leading front position of an eruption and the center of the Sun can be corrected for the projection effect. The real distance, $d_{\mathrm{r}}$, can be calculated from the following equation (after including Eq. (A.1))

$d_{\mathrm{r}}=\frac{d_{\mathrm{a}}}{\sin \delta}=\frac{d_{\mathrm{a}}}{\left(1-\cos ^{2} \lambda^{\prime} \cos ^{2} \phi^{\prime}\right)^{2}}$,

finally, subtracting $R_{\odot}$ from the distance $d_{\mathrm{r}}$, the real height, $h$, of an eruption above the photosphere can be obtained: $h=d_{\mathrm{r}}-R_{\odot}$.

The real height uncertainty is caused mainly by uncertainties in measurement of the positions of the eruption leading front and the reference point. We calculated the error on the height, $h$, using the standard propagation of uncertainty methods.

\section{References}

Amari, T., \& Luciani, J. F. 1999, ApJ, 515, L81

Antiochos, S. K., \& Sturrock, P. A. 1978, ApJ, 220, 1137

Archontis, V., \& Török, T. 2008, A\&A, 492, L35

Aschwanden, M. J., Newmark, J. S., Delaboudinière, J.-P., et al. 1999, ApJ, 515, 842

Gburek, S., Sylwester, J., \& Martens, P. 2006, Sol. Phys., 239, 531

Gilbert, H. R., Alexander, D., \& Liu, R. 2007, Sol. Phys., 245, 287

Green, L. M., Matthews, S. A., van Driel-Gesztelyi, L., Harra, L. K., \& Culhane, J. L. 2002, Sol. Phys., 205, 325

Handy, B. N., Acton, L. W., Kankelborg, C. C., et al. 1999, Sol. Phys., 187, 229
Hirose, S., Uchida, Y., Uemura, S., Yamaguchi, T., \& Cable, S. B. 2001, ApJ, 551,586

Hurford, G. J., Schmahl, E. J., Schwartz, R. A., et al. 2002, Sol. Phys., 210, 61

Kontar, E. P., \& MacKinnon, A. L. 2005, 227, 299

Kosugi, T., Masuda, S., Makishima, K., et al. 1991, Sol. Phys., 136, 17

Lin, A. C., Nightingale, R. W., \& Tarbell, T. D. 2001, Sol. Phys., 198, 385

Lin, R. P., Dennis, B. R., Hurford, G. J., et al. 2002, Sol. Phys., 210, 3

Liu, Y. 2008, ApJ, 679, L151

Liu, R., \& Alexander, D. 2009, ApJ, 697, 999

Metcalf, T. R., Hudson, H. S., Kosugi, T., Puetter, R. C., \& Pina, R. K. 1996, ApJ, 466, 585

Mrozek, T. 2011, Sol. Phys., 270, 191

Mrozek, T., \& Tomczak, M. 2004, A\&A, 415, 377

Mrozek, T., Tomczak, M., \& Gburek, S. 2007, A\&A, 472, 945

Phillips, K. J. H., Chifor, C., \& Landi, E. 2005, ApJ, 626, 1110

Sakai, J.-I., \& de Jager, C. 1996, Space Sci. Rev., 77, 1

Shen, Y.-D., Liu, Y., \& Liu, R. 2011, Res. Astron. Astrophys., 11, 594

Shibata, K. 1999, Ap\&SS, 264, 129

Smith, D. M., Lin, R. P., Turin, P., et al. 2002, Sol. Phys., 210, 33

Temmer, M., Veronig, A. M., Kontar, E. P., Krucker, S., \& Vršnak, B. 2010, ApJ, 712,1410

Tomczak, M. 1999, A\&A, 342, 583

Török, T., \& Kliem, B. 2005, ApJ, 630, L97

Uchida, Y., Hirose, S., Cable, S., et al. 1999, PASJ, 51, 553

Vršnak, B. 1990, Sol. Phys., 129, 295

Vršnak, B. 2001, J. Geophys. Res., 106, 25249

Wang, Y., \& Zhang, J. 2007, ApJ, 665, 1428

Wang, Y.-M., \& Sheeley, Jr., N. R. 1992, ApJ, 392, 310

Warren, H. P., Winebarger, A. R., \& Hamilton, P. S. 2002, ApJ, 579, L41

Wen, Y., Maia, D. J. F., \& Wang, J. 2007, ApJ, 657, 1117

Winebarger, A. R., Warren, H. P., \& Seaton, D. B. 2003, ApJ, 593, 1164 